\title{
Manejo nutricional na constipação intestinal em terapia de nutrição enteral: um guia de orientações
}

\author{
Nutritional management in intestinal constipation enteral nutrition therapy: a guidance guide \\ Manejo nutricional en el estreñimiento intestinal en terapia de nutrición enteral: una guía de \\ orientación
}

Recebido: 16/01/2022 | Revisado: 21/01/2022 | Aceito: 01/02/2022 | Publicado: 03/02/2022

\author{
Laila dos Santos Morais \\ ORCID: https://orcid.org/0000-0002-2464-7787 \\ Centro Universitário Uninovafapi, Brasil \\ E-mail: lailamoraisnutricionista@gmail.com \\ Leticia Maria Sampaio Silva \\ ORCID: https://orcid.org/0000-0002-0479-4587 \\ Centro Universitário Uninovafapi, Brasil \\ E-mail: leticiasampaionutricionista@gmail.com \\ Nataline Rodrigues Nunes \\ ORCID: https://orcid.org/0000-0002-8397-3902 \\ Centro Universitário Uninovafapi, Brasil \\ E-mail: naty20nunes@hotmail.com \\ Vania Marisa da Silva Vasconcelos \\ ORCID: https://orcid.org/0000-0003-1795-7725 \\ Centro Universitário Uninovafapi, Brasil \\ E-mail: vania.vasconcelos@uninovafapi.edu.br \\ Alessandro Carvalho Alves dos Santos \\ ORCID: https://orcid.org/0000-0002-6925-643X \\ Centro Universitário Uninovafapi, Brasil \\ E-mail: alessandro.santos@uninovafapi.edu.br \\ Layana Rodrigues Chagas \\ ORCID: https://orcid.org/0000-0002-2930-8899 \\ Centro Universitário Uninovafapi, Brasil \\ E-mail: layana_rodrigues@yahoo.com.br \\ Norma Sueli Marques Da Costa Alberto \\ ORCID: https://orcid.org/0000-0002-9412-0817 \\ Centro Universitário Uninovafapi, Brasil \\ E-mail: normaalberto@yahoo.com.br
}

\begin{abstract}
Resumo
Objetivo: Descrever a elaboração de um guia de manejo nutricional para constipação intestinal (CI) na Terapia de Nutrição Enteral (TNE). Método: Estudo metodológico que descreve a elaboração de uma tecnologia educacional de apoio à conduta terapêutica, realizado em três etapas: levantamento bibliográfico nas bases acadêmicas: biblioteca virtual de saúde (BVS), Scielo e Google Acadêmico, acerca da etiopatogenia da síndrome da CI; identificação de insumos dietéticos enterais, com sua composição e indicação de uso em CI, disponíveis no mercado nacional, por meio de buscas em sites; e, última etapa, a confecção do guia de manejo nutricional. Resultados e discussão: O guia apresenta: capa, contracapa, apresentação, sumário, introdução, manejo nutricional e informações relevantes ao entendimento da CI, quadros informativos de insumos dietéticos disponíveis no mercado nacional com indicação de uso em constipação intestinal, fechamento e referências. Foram incluídos no guia, 11 fórmulas com indicação para a CI e para os transtornos gastrointestinais e 23 insumos suplementares, totalizando 34 produtos enterais com alegação para a CI, disponíveis no mercado, e ressaltando, quanto às fibras, a pectina e a goma guar, como de maior predominância na nutrição enteral, e que mais auxiliam na função intestinal em TNE por períodos prolongados. Conclusão: O guia possibilita aos profissionais da área da saúde, sobretudo nutricionistas, um manejo adequado da CI, trazendo informações relevantes e sugestões de insumos dietéticos para uso em TNE.
\end{abstract}

Palavras-chave: Terapia nutricional; Nutrição enteral; Constipação intestinal; Fibras alimentares.

\begin{abstract}
Objective: This study aimed to develop a guide of nutritional guidelines for intestinal constipation in Enteral Nutrition Therapy (ENT). Methods: Methodological development study, which describes the elaboration of an educational technology to support the therapeutic conduct. The research was carried out in three stages: The first stage consisted of a bibliographic survey in the academic databases virtual health library (VHL), Scielo and Google Scholar about the
\end{abstract}


etiopathology of the syndrome; The second step was the search for enteral products, through websites, to identify dietary inputs and their composition, with indication for use in intestinal constipation, available in the national market. The third was the preparation of the nutritional management guide. Results and discussion: The guide presents: cover, back cover, presentation, summary, introduction, nutritional management and relevant information to the understanding of IC, information tables of dietary supplies available in the national market with indication for use in constipation, closing, and references. The guide included 11 formulas indicated for IC and for gastrointestinal disorders and 23 supplementary supplies, totaling 34 enteral products with claims for IC8, available on the market, and highlighting, as to fibers, pectin and guar gum, as the most predominant in enteral nutrition, and that most assist intestinal function in TNE for prolonged periods. Conclusion: The guide enables nutrition professionals to adequately manage constipation, providing relevant information about IC and suggestions for dietary inputs for use in ENT.

Keywords: Nutritional therapy; Nutrition enteral; Constipation; Foodfibers.

\section{Resumen}

Objetivo: Desarrollar una guía de manejo nutricional para el estreñimiento en la Terapia de Nutrición Enteral (ENT). Metodología: Estudio de desarrollo metodológico, que describe el desarrollo de una tecnología educativa para apoyar la conducta terapéutica. La investigación se llevó a cabo en tres etapas: La primera etapa consiste en un levantamiento bibliográfico en las bases de datos académicas Biblioteca Virtual de Salud (BVS), Scielo y Google Académico sobre la etiopatología del síndrome; la segunda etapa fue la búsqueda de productos enterales, a través de sitios web, para identificar insumos dietéticos y su composición, con indicación para uso en estreñimiento intestinal, disponibles en el mercado nacional. El tercero fue la creación de la guía de manejo nutricional. Resultados y discusión: La guía presenta: portada, contraportada, presentación, resumen, introducción, manejo nutricional e información relevante para la comprensión de la CI, tablas informativas de suministros dietéticos disponibles en el mercado nacional con indicación de uso en el estreñimiento, cierre y referencias. La guía incluyó 11 fórmulas indicadas para el estreñimiento y para los trastornos gastrointestinales y 23 complementos, totalizando 34 productos enterales con alegaciones para el estreñimiento, disponibles en el mercado, y destacando, en cuanto a las fibras, la pectina y la goma guar, como las más predominantes en la nutrición enteral, y las que más ayudan a la función intestinal en la ENT durante periodos prolongados. Conclusión: La guía permite a los profesionales de la salud manejar adecuadamente el estreñimiento, brindando información relevante sobre CI y sugerencias de insumos dietéticos para uso en ORL.

Palabras clave: Terapia nutricional; Nutrición enteral; Estreñimiento; Fibras alimentarias.

\section{Introdução}

A nutrição enteral é definida como alimento para fins especiais, com ingestão controlada de nutrientes, na forma isolada ou combinada, de composição definida ou estimada, especialmente formulada para uso por sondas ou via oral (Barros et al., 2019). É utilizada para indivíduos sem condições de deglutir ou quando não consegue atingir pelo menos $60 \%$ de suas necessidades nutricionais diárias por via oral, visando melhorar as condições nutricionais dos mesmos (Cervo et al., 2014).

A Terapia nutricional enteral (TNE) é caracterizada por procedimentos terapêuticos com função de recuperar ou manter o estado nutricional dos indivíduos (Oliveira, 2021). Alguns estudos apontam que a oferta da TNE apresenta fatores que podem limitar a administração adequada de nutrientes, resultando em situações de disfunção do trato gastrointestinal, tais como: náuseas, vômito, diarreia, distensão abdominal e Constipação Intestinal (CI) (Shils, 2003).

A CI é definida como eliminação de fezes menor que três vezes por semana, sensação de esvaziamento retal incompleto, fezes endurecidas e esforço para a evacuação (SBCP, 2009).

Estudos realizado por Guerra (2013) apontam que 69\% dos pacientes em TNE são acometidos com a CI. O que pode ser decorrente da ausência de fibras na dieta enteral, da vulnerabilidade nutricional, do tempo de permanência hospitalar, dos sintomas gastrointestinais e da instabilidade do paciente a dieta (Melo et al.,2017)

Em alguns casos, pacientes que apresentam CI permanecem internados por mais tempo, em média oito dias, impactando diretamente na sua evolução, uma vez que ficam expostos a outras infecções, translocação bacteriana, bem como as próprias complicações da CI como distensão abdominal, vômitos, agitação, obstrução intestinal e perfuração intestinal (Barros, 2019). 
Os tratamentos propostos são terapias não farmacológicas como: mudanças na dieta, ingestão hídrica adequada e consumo de fibras; e farmacológicas, sendo os laxantes os medicamentos mais prescritos que, quando utilizados de forma abusiva, podem desequilibrar a microbiota intestinal saudável (Júnior, 2005; Almeida et al., 2009).

Nesse contexto é importante ressaltar que uma microbiota equilibrada promove o bem-estar e a ausência de doenças, especialmente do trato gastrintestinal, e para mantê-la saudável é necessário uso de alimentos funcionais como: prebióticos, probióticos e simbióticos (Isolauri, 2004; Santos, 2011).

Os prebióticos, fibras não digeríveis pelo nosso organismo, são totalmente utilizados pelas bactérias probióticas e sua ação estimula seletivamente a proliferação ou atividade de populações de bactérias desejáveis no cólon, sendo responsável pela manutenção dessas bactérias (Ferreira, 2014). Já os simbioticos são a combinação de um probióticos com um prebiótico, e o uso deste otimiza o sistema imunológico intestinal favorecendo o controle da microbiota e diminuindo a incidência de infecções do trato gastrointestinal (Ferreira, 2012).

Diante da frequente ocorrência da CI, da relevância dos impactos que causa, bem como da dificuldade de se estabelecer os fatores etiológicos e manejos adequados para solucionar a situação clínica (Reis et al., 2018), são necessárias estratégias intervenientes de manejos adequados para prevenir ou tratar esta condição clínica.

A partir desse pressuposto sucedeu-se a elaboração de um Guia, ferramenta que apresenta uma fundamentação teórica- metodológica, possibilitando o repasse de informações ao leitor de forma clara e objetiva (Silva, 2014), visto que as ferramentas de tecnologias educacionais são alternativas que podem propagar a interação da pesquisa e informação no meio social (Maciel, 2002).

Nesse sentido este estudo teve por objetivo descrever a elaboração de um guia de orientações nutricionais para o manejo da CI a TNE que possa subsidiar profissionais da área da saúde, sobretudo os nutricionistas, em suas condutas.

\section{Metodologia}

Estudo metodológico, que descreve de forma pormenorizada a elaboração dos instrumentos e técnicas de pesquisa, cujo objetivo é a construção de um produto que seja confiável, preciso e utilizável para que possa ser disponibilizado a outros pesquisadores (Santana, 2012).

Dessa forma, foi realizada a elaboração de um guia de manejo nutricional na constipação intestinal em adultos e idosos em uso de TNE. O estudo foi desenvolvido, no período de setembro a novembro de 2021, através de pesquisa pela internet para levantamento bibliográfico de informações sobre constipação intestinal, e identificação de insumos dietéticos.

O estudo foi realizado em três etapas: A primeira foi um levantamento bibliográfico nas bases acadêmicas: Biblioteca Virtual de Saúde (BVS), Scielo e Google Acadêmico acerca da etiopatogenia e tratamento da constipação intestinal, bem como conceitos de fibras, probióticos, prebióticos e simbióticos para compor um guia com informações relevantes e necessárias ao manejo nutricional na CI sendo considerados os artigos publicados a partir de 2001 nos idiomas português e inglês. Utilizou-se os seguintes descritores: Terapia nutricional enteral, Constipação intestinal e fibras alimentares, os artigos que apresentavam apenas terapia medicamentosa e/ou terapias alternativas incompatíveis com uso por nutrição enteral foram excluídos.

A segunda etapa ocorreu pela busca de insumos dietéticos, em sites e aplicativos de empresas especializadas em produtos para nutrição enteral, disponíveis no mercado nacional, indicados para CI, para auxiliar no funcionamento do trato gastrointestinal, contendo fibras e/ou probióticos, sendo identificados os tipos e os teores de fibras, bem como os tipos de produtos (fórmulas completas e/ou suplementos nutricionais). Foram pesquisados produtos que podem ser utilizados com pacientes tanto a nível hospitalar, quanto domiciliar.

A terceira etapa sucedeu a confecção do Guia de Orientações Nutricionais para Constipação Intestinal em TNE, 
composto por: capa, contracapa, apresentação, sumário, introdução, informações relevantes ao entendimento e manejo nutricional da Constipação Intestinal e ilustrações de insumos dietéticos disponíveis no mercado nacional com indicação de uso em constipação intestinal, fechamento e referências. Quanto à estrutura e apresentação, a arte do guia foi desenvolvida por desing gráfico, em tamanho de papel A5 $(148 \times 210 \mathrm{~mm})$, fonte arial, tamanho 10 , com cores pretas para a fonte no corpo do texto e azul para títulos.

\section{Resultados e Discussão}

Segundo Barbosa, Colares e Soares (2008) os guias devem ser elaborados, com termos que sejam compreensíveis, simples e claros para a maioria dos consumidores e indicando as informações necessárias inerentes ao conteúdo ofertado. A partir dessa premissa, elaborou-se o conteúdo de forma bem sucinta, objetiva e ilustrativa, de modo a facilitar o entendimento e visualização por parte dos usuários. A seguir, as ilustrações mostram como ficou a versão final do guia impresso.

\subsection{Capa e contra capa. (Figura 1)}

Na capa foi colocado o titulo "Manejo nutricional na Constipação Intestinal em Terapia de Nutrição Enteral" e na contra capa os nomes dos autores e colaboradores da elaboração e catálogo de publicação.

Figura 1 - Ilustração representativa da Capa, e contra capa.

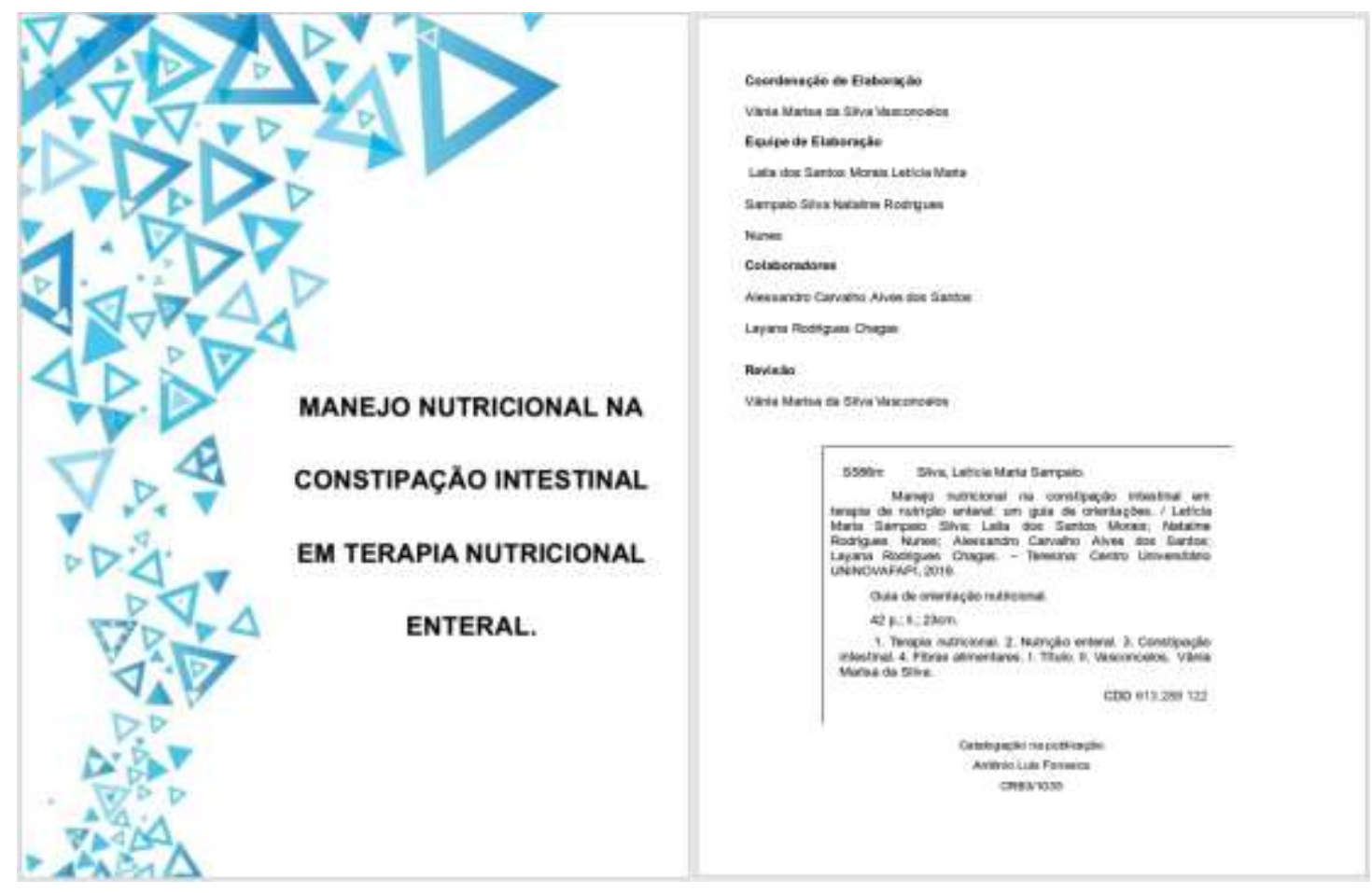

Fonte: Autores.

\subsection{Apresentação e Sumário. (Figura 2)}

No capitulo correspondente à apresentação, apresentou-se de forma sucinta o guia, abordando a origem, finalidade e qual público é destinado. Já no sumário contém a identificação de todo o conteúdo do guia e o número de páginas correspondente. 
Figura 2 - Ilustração representativa da apresentação e Sumário.

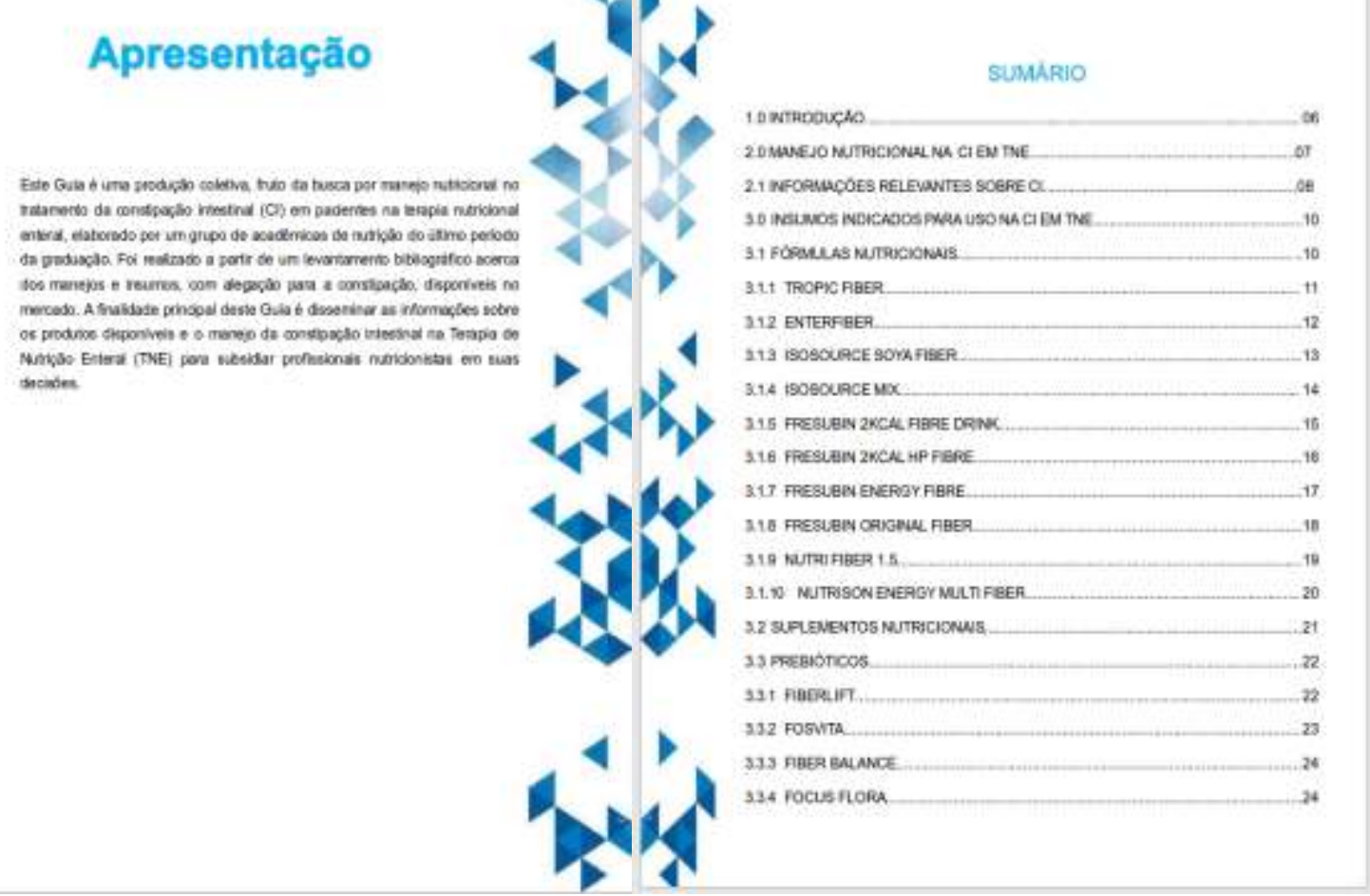

Fonte: Autores.

\subsection{Introdução, Manejo e Informações relevantes para a CI. (Figura 3)}

Segundo Deheinzelin (2012), a introdução é a forma mais concisa e objetiva para a comunicação científica, por isso deve ser referenciada, com o intuito de propor informações fidedignas de forma clara, objetiva e fundamentada, proporcionando ao leitor o entendimento do assunto em questão. Pensando nisso, o capítulo introdutório foi abordado os conceitos de nutrição enteral e CI, assim como sua incidência em pessoas que estão em uso de TNE.

No capítulo que trata das informações relevantes, explorou-se os tratamentos propostos, onde se ressaltou a importância das fibras em pessoas acometidas com CI. Elas são classificadas em solúveis e insolúveis, as da categoria insolúvel como: lignina, celulose e algumas hemiceluloses, são viscosas e facilmente fermentáveis no intestino grosso, representando as mais benéficas no tratamento da obstipação, uma vez que retém maior quantidade de água e, desse modo, aumentam a massa fecal e estimulam os movimentos peristálticos, diminuindo o tempo de trânsito intestinal (Montejo et al., 2001).

Tendo em vista que as fibras desempenham tantos papéis essenciais ao bom funcionamento do intestino, além de outras inúmeras funções no organismo como um todo. Elas atuam na prevenção e no tratamento da CI, especialmente as do tipo insolúveis as quais contribuem com a formação, amolecimento e eliminação do bolo fecal. Além disso, explicou-se também a relevância dos compostos: prebióticos, probióticos e simbióticos na promoção da saúde do intestino como um todo e na melhora em quadros de CI. 
Figura 3 - Ilustração representativa da Introdução, manejo e informações relevantes.

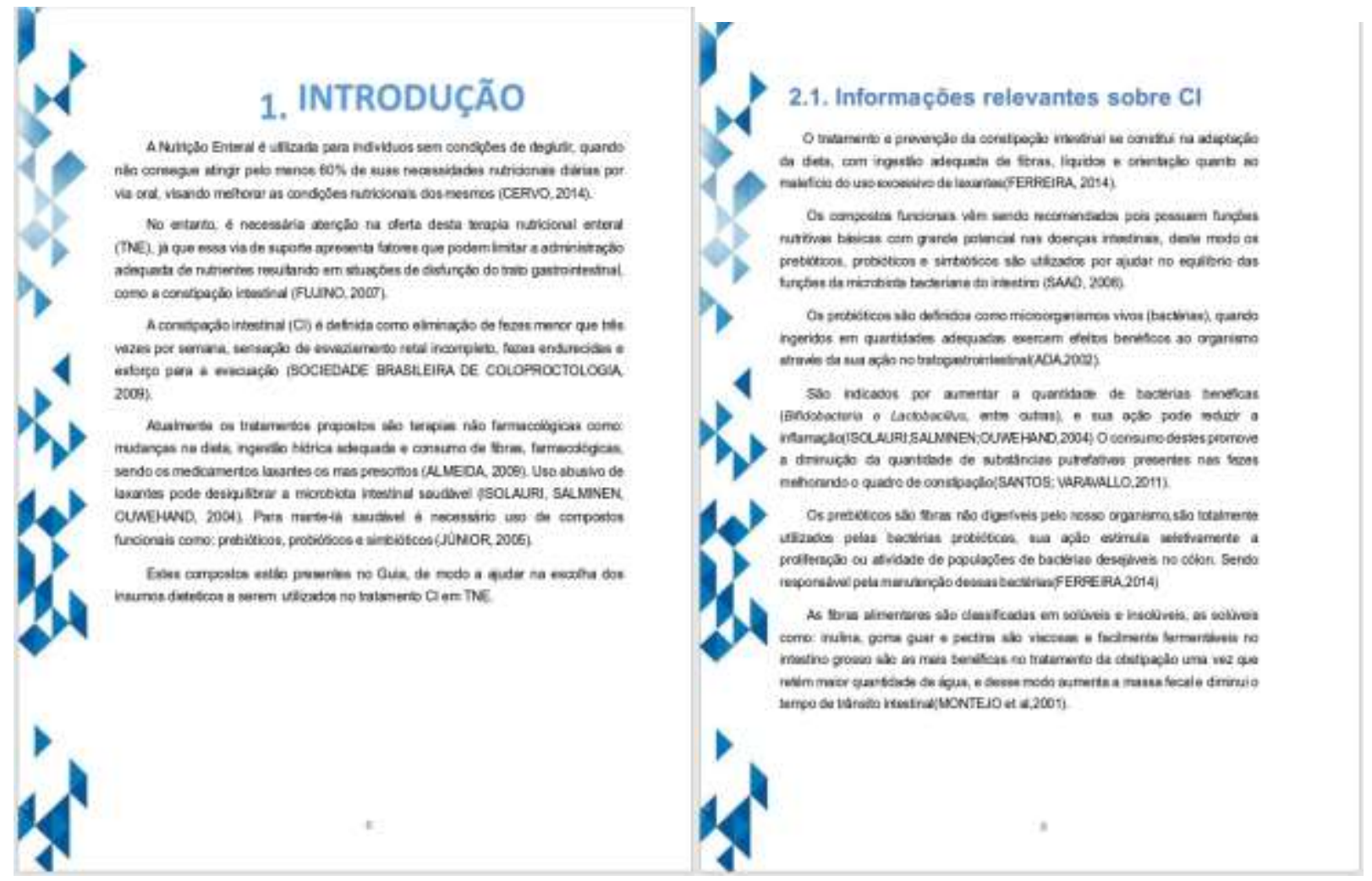

Fonte: Autores.

\subsection{Insumos dietéticos para uso na CI via Nutrição Enteral. (Figura 4)}

Neste capítulo o Guia traz de modo ilustrativo as fórmulas nutricionais enterais e suplementos nutricionais, mostrando a forma de apresentação, indicação de uso, laboratório responsável pela formulação e composição nutricional, observou-se que os produtos não possuem contra indicação, e caso contrário, não foi especificado pelo fabricante na rotulagem. Após as ilustrações, foram inseridos quadros com todas as fórmulas e suplementos utilizados no guia. Por meio dessas orientações e informações, o profissional de saúde pode conhecer, compreender o produto e identificar qual o melhor para a necessidade do paciente em questão. 
Research, Society and Development, v. 11, n. 2, e48011225801, 2022

(CC BY 4.0) | ISSN 2525-3409 | DOI: http://dx.doi.org/10.33448/rsd-v11i2.25801

Figura 4 - Ilustração representativa das formulas nutricionais.

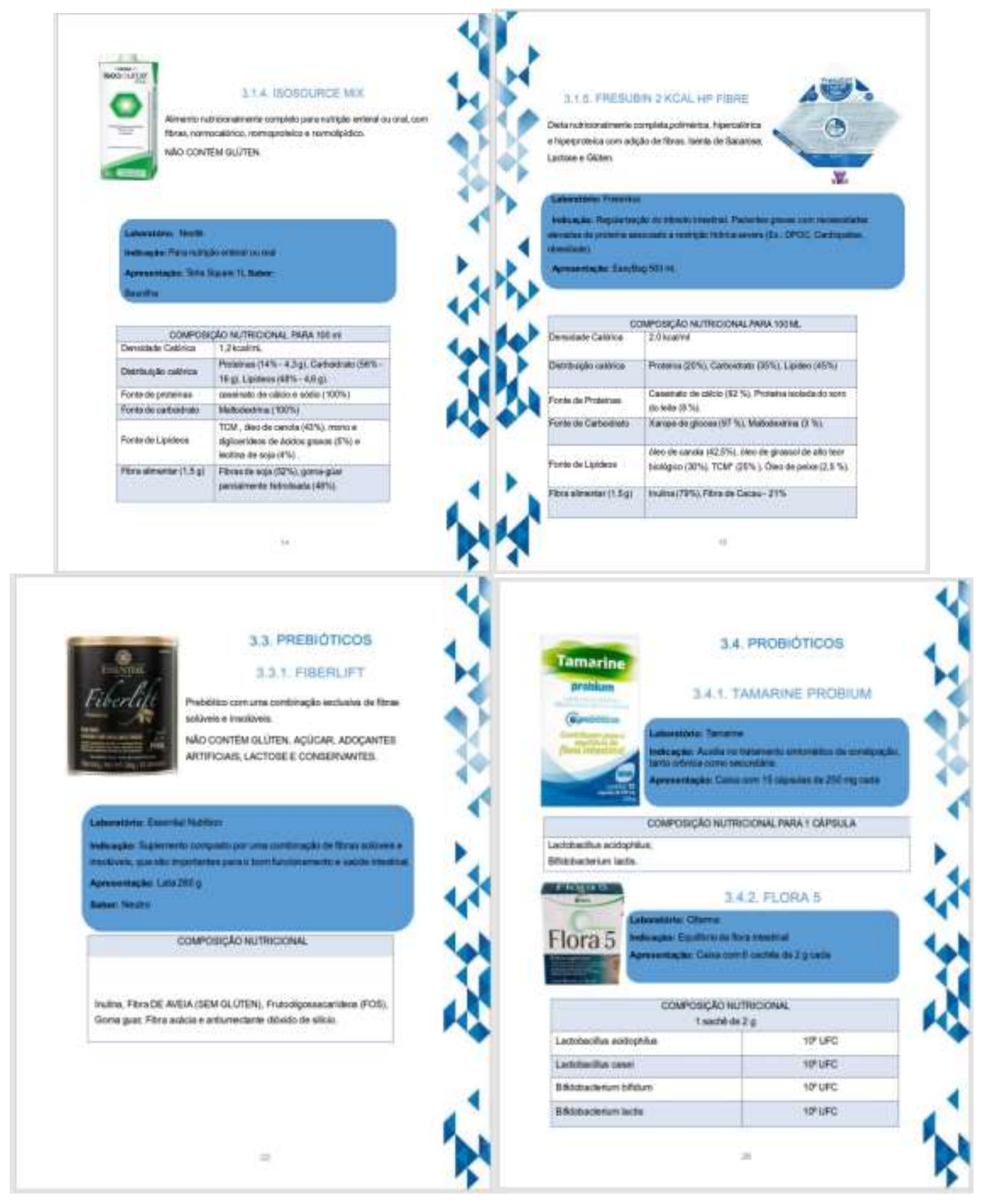

Fonte: Autores. 
Research, Society and Development, v. 11, n. 2, e48011225801, 2022

(CC BY 4.0) | ISSN 2525-3409 | DOI: http://dx.doi.org/10.33448/rsd-v11i2.25801

Quadro 1: Fórmulas nutricionais com indicação para Constipação Intestinal TNE. Teresina, 2021.

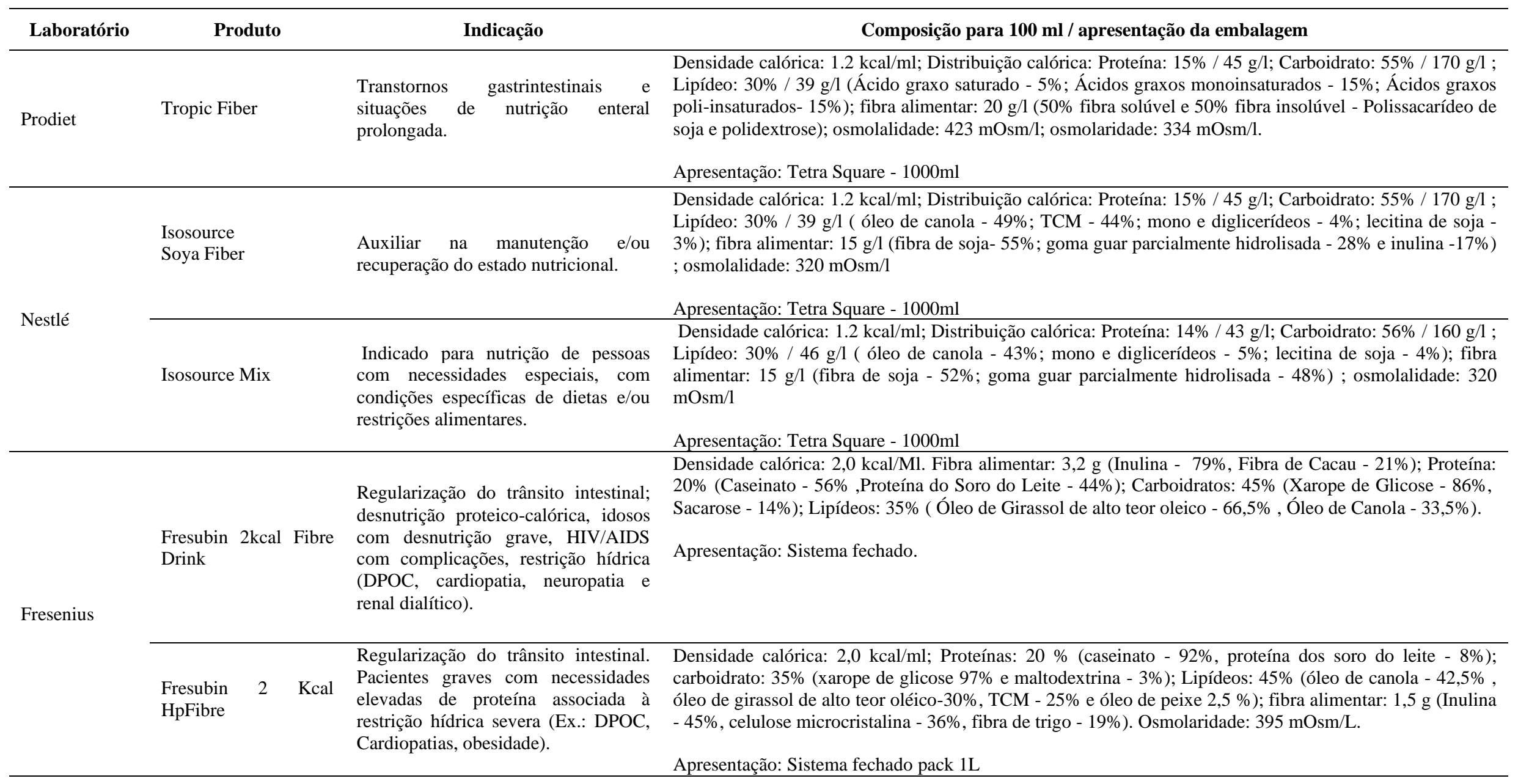


Research, Society and Development, v. 11, n. 2, e48011225801, 2022

(CC BY 4.0) | ISSN 2525-3409 | DOI: http://dx.doi.org/10.33448/rsd-v11i2.25801

Fresubin Energy Fibre Regularização do trânsito intestinal. bucomaxilo. Risco nutricional ou desnutrição leve,
idosos frágeis, cirurgia de

Densidade calórica: 1,5 kcal/ mL. Fibra alimentar: 4,0 g (Fibra de Trigo - 41,5 b e 46,5\%, Inulina - 33,5\%, Celulose Microcristalina - 2 b e 20\%, Fibra de Cacau - 23\% b);Proteínas: $15 \%$ ( Caseinato - 86\%, Proteína do Soro do Leite - 14\%); Carboidratos: 50\% (Maltodextrina - 69 a 74,6\% a, Sacarose - 25,4 a 31\% a); Lipídeos: 35\% (Óleo de Canola - 75\%, Óleo de Girassol de alto teor oléico 25\%).

Apresentação: Sistema fechado pack 1L

\section{Densidade Calórica:1,0 kcal/ml; Proteínas: $15 \%$ (51\% caseinato e $49 \%$ Proteína isolada de soja);} carboidratos:55\%(maltodextrina); Lipídeos: $30 \%$ (73\% óleo de canola, $24 \%$ leo de girassol de alto teor oléico e $3 \%$ de óleo de peixe); fibra alimentar: 1,5 g (45\% Inulina, 38\% celulose microcristalina e 17\% fibra de trigo); osmolaridade: $285 \mathrm{mOsm} / \mathrm{L}$

Apresentação: Sistema fechado pack 1L

Densidade Calórica: $1.5 \mathrm{kcal} / \mathrm{ml}$; Proteínas:17\% (Prot. do Soro do Leite - 60\%, Caseinato de Cálcio e Sódio - 40\%), Carboidratos: 58\% (Maltodextrina - 100\%), Lipídios: 25\% (Óleo de Girassol - 12\%, Óleo de Canola-79\%, TCM-9\%).

Apresentacão: Tetra Square - 1000ml

Densidade calórica: $1.5 \mathrm{kcal} / \mathrm{ml}$; Carboidrato: $49 \%$ (Maltodextrina -100\%); Proteína: $16 \%$ (Concentrado proteico do soro do leite $-35 \%$, Caseinato de sódio $-25 \%$, Proteína Isolada de ervilha $-20 \%$, Proteína Isolada de soja $-20 \%$ ); Lipídeos: $36 \%$ (Óleo de canola $-37,9 \%$, Óleo de girassol $-42,9 \%$, TCM $-17,5 \%$, Óleo de peixe $-1,7 \%)$; Isenta de lactose, sacarose e glúten; fibras solúveis $(80 \%)$ e insolúveis $(20 \%)$

Apresentação: sistema fechado pack 1L

Densidade calórica: $1.0 \mathrm{kcal} / \mathrm{ml}$; Distribuição calórica: Proteína: 17\% / 42 g/l; Carboidrato: 33\% / $18 \mathrm{~g} / \mathrm{l}$; Lipídeo: $50 \%$ / $54 \mathrm{~g} / 1$ ( Óleo de girassol - 85\%; Óleo de canola: 10\%; Lecitina - 5\%); fibra alimentar: Polissacarídeo de soja - $14 \mathrm{~g} / \mathrm{l}$ osmolalidade: $354 \mathrm{mOsm} / \mathrm{l}$; osmolaridade: $300 \mathrm{mOsm} / \mathrm{l}$.

Glucerna RTH para paciente com diabetes tipo 1 e 2 , que precisam de suplementação para controle da glicemia; ajuda na regulação intestinal

Apresentação: Sistema fechado1000mL
Abbott

Jevity Plus RTH
Densidade calórica: 1.2 kcal/ml; Distribuição calórica: Proteína: 18,5\% / 55,5 g/l; Carboidrato: 52,5\% / 151 g/l ; Lipídeo: 29\% / 39 g/l ( Óleo de açafrão de alto teor biológico - 47,5\%; Óleo de canola: 28,5\%; TCM Indicado para pacientes com 19\%; Lecitina - 5\%); fibra alimentar: $22 \mathrm{~g} / \mathrm{l}$ (FOS - $10 \mathrm{~g} / 1$; Combinação de fibras - fibra de soja, fibra de necessidade de normalização do aveia e goma arábica - $12 \mathrm{~g} / \mathrm{l})$; osmolalidade: $450 \mathrm{mOsm} / \mathrm{l}$; osmolaridade: $365 \mathrm{mOsm} / 1$

trânsito intestinal, mas com restrição a grandes volumes
Apresentação: sistema fechado $1000 \mathrm{~mL}$ 
Research, Society and Development, v. 11, n. 2, e48011225801, 2022

(CC BY 4.0) | ISSN 2525-3409 | DOI: http://dx.doi.org/10.33448/rsd-v11i2.25801

Quadro 2: Suplementos/módulos nutricionais com indicação para Constipação Intestinal TNE. Teresina, 2021.

\begin{tabular}{|c|c|c|c|}
\hline Tipo de insumos & Produto/ laboratório & Indicação & Composição / apresentação da embalagem \\
\hline \multirow[t]{10}{*}{ Prebiótico } & $\begin{array}{l}\text { Fiber lift } \\
\text { (Essential Nutrition) }\end{array}$ & $\begin{array}{l}\text { Suplemento composto por uma combinação de } \\
\text { fibras solúveis e insolúveis, que são importantes } \\
\text { para o bom funcionamento e saúde intestinal. }\end{array}$ & $\begin{array}{l}\text { Inulina, fibra natural, frutooligossacarídeos (FOS), goma guar e polidextrose. } \\
\text { Fibra alimentar: } 4,5 \mathrm{~g} \\
\text { Apresentação: pó }\end{array}$ \\
\hline & $\begin{array}{l}\text { Enter } \\
\text { (laboratório?) }\end{array}$ & Transtornos gastrintestinais. & $\begin{array}{l}\text { Fibra alimentar: } 8,3 \text { g (65\% Fibras Solúveis e } 35 \% \text { Fibras Insolúveis; Polidextrose e } \\
\text { Polissacarídeo de Soja); Carboidrato: 4,8g; Proteínas: 4,4g; } \\
\text { Apresentação: pó }\end{array}$ \\
\hline & $\begin{array}{l}\text { Natural Fiber } 3 \\
\text { (Nutrata Nature) }\end{array}$ & $\begin{array}{l}\text { Estimula o trânsito intestinal e previne a } \\
\text { colonização de bactérias patogênicas }\end{array}$ & $\begin{array}{l}\text { Inulina, fibra de acácia, goma guar parcialmente hidrolisada. } \\
\text { Fibra: } 5,7 \mathrm{~g} \\
\text { Apresentação: pó (lata - 200g) }\end{array}$ \\
\hline & $\begin{array}{l}\text { Stimulance multifiber } \\
\text { (Danone) }\end{array}$ & $\begin{array}{l}\text { Contribui para a regularização do trânsito } \\
\text { intestinal, minimizando os casos de diarreia e } \\
\text { constipação. Auxilia no controle de doenças } \\
\text { como diabetes, hipercolesterolemia, } \\
\text { diverticulite, etc }\end{array}$ & $\begin{array}{l}\text { Polissacarídeos de soja, inulina, amido resistente, goma arábica, oligiofrutose(FOS), celulose. } \\
\text { Fibra: } 76 \mathrm{~g} \\
\text { Apresentação: pó (lata- 225g) }\end{array}$ \\
\hline & Fosvita (Vitafor) & Auxilia no funcionamento do intestino. & $\begin{array}{l}\text { Frutooligossacarideos em pó. Fibra } 7 \mathrm{~g} \text {. } \\
\text { Apresentação: sachê de } 10 \mathrm{~g}\end{array}$ \\
\hline & $\begin{array}{l}\text { Fiber } \\
\text { (Nutrify) }\end{array}$ & Saúde intestinal. & $\begin{array}{l}\text { Goma guar parcialmente hidrolisada, inulina e dióxido de silício (antiumectante). Fibra: } 4,4 \mathrm{~g} \\
\text { Apresentação: Lata } 200 \mathrm{~g}\end{array}$ \\
\hline & Focus Flora (ADS) & $\begin{array}{l}\text { Equilíbrio da flora intestinal; inibe o crescimento } \\
\text { de bactérias prejudiciais ao organismo. }\end{array}$ & $\begin{array}{l}\text { Fruto-oligossacarídeos (FOS). Fibra: } 6 \mathrm{~g} \\
\text { Apresentação: pó }\end{array}$ \\
\hline & Fiber Mais (Nestlé) & $\begin{array}{l}\text { Auxiliam o funcionamento do intestino de } \\
\text { maneira regular e saudável e promove o } \\
\text { equilíbrio da flora intestinal }\end{array}$ & $\begin{array}{l}\text { Goma Guar Parcialmente Hidrolisada e Inulina. } \\
\text { Apresentação: lata de } 260 \mathrm{~g} \text { ou caixa com } 10 \text { sachês de } 5 \mathrm{~g} \text {. }\end{array}$ \\
\hline & $\begin{array}{l}\text { Fiber } \\
\text { (Sanativa) }\end{array}$ & $\begin{array}{l}\text { Destinada a indivíduos que buscam uma dieta } \\
\text { rica em fibras. }\end{array}$ & $\begin{array}{l}\text { Mix de } 7 \text { fibras alimentares solúveis e insolúveis: polidextrose, amido resistente, celulose, } \\
\text { inulina, fibra de aveia, goma arábica e fibra de maçã. Fibra: } 4 \mathrm{~g} \\
\text { Apresentação: lata de } 225 \mathrm{~g} \text { com colher medida de } 5 \mathrm{~g} \text { ou caixa com } 10 \text { sachês de } 5 \mathrm{~g} \text {. }\end{array}$ \\
\hline & $\begin{array}{l}\text { Florastor } \quad \text { (União } \\
\text { Química) }\end{array}$ & Equilibra e restaura a flora intestinal. & $\begin{array}{l}\text { Lactobacillus acidophilus. } \\
\text { Apresentação: sachê de } 4 \mathrm{~g} .\end{array}$ \\
\hline \multirow[t]{3}{*}{ Probiótico } & Flora5 (Cifarma) & Equilíbrio da flora intestinal & $\begin{array}{l}\text { Lactobacillusacidophilus, Lactobacillus casei }\left(10^{9} \mathrm{UFC}\right) ; \text { Bifidobacteriumbifidum }\left(10^{9} \mathrm{UFC}\right) \text {; } \\
\text { Bifidobacterium lactis }\left(10^{9} \mathrm{UFC}\right) \text {. } \\
\text { Apresentação: sachê de } 2 \mathrm{~g} \text { cada }\end{array}$ \\
\hline & Valence (Baldacci) & Equilíbrio da flora intestinal & $\begin{array}{l}\text { Bifidobacterium lactis } 10^{9} \text { UFC } \\
\text { Apreentação: sachê de } 2 \mathrm{~g}\end{array}$ \\
\hline & Bio 5 cepas (Genom) & Contribui para o equilíbrio da flora intestinal & $\begin{array}{l}\text { Bifidobacterium lactis/ Lactobacillus rhamnosus/ Lactobacillus casei/ Lactobacillus paracasei / } \\
\text { Lactobacillus } \\
\text { Apresentação: sachê de } 4 \mathrm{~g}\end{array}$ \\
\hline
\end{tabular}


Research, Society and Development, v. 11, n. 2, e48011225801, 2022

(CC BY 4.0) | ISSN 2525-3409 | DOI: http://dx.doi.org/10.33448/rsd-v11i2.25801

\begin{tabular}{|c|c|c|c|}
\hline & Simfort (Vitafor) & Contribui para uma flora intestinal saudável. & $\begin{array}{l}\text { Lactobacillusacidophilus }\left(10^{9} \text { UFC), Lactobacillus casei }\left(10^{9} \text { UFC), Lactobacilluslactis }\left(10^{9}\right.\right.\right. \\
\text { UFC), Bifidobacteriumbifidum }\left(10^{9} \text { UFC), Bifidobacteriumlactis }\left(10^{9} \text { UFC }\right)\right. \\
\text { Apresentação: sachê de } 2 \mathrm{~g}\end{array}$ \\
\hline & Bidrilac (Daudt) & $\begin{array}{l}\text { Tratamento da Flora Intestinal, Processos } \\
\text { Inflamatórios da Boca e Garganta, Diarreia, Dor } \\
\text { de Barriga, Obstipação Intestinal, Problemas no } \\
\text { Intestino, Intestino Preso, Prisão de Ventre. }\end{array}$ & $\begin{array}{l}\text { Lactobacillus acidophilus LA-5 (10 UFC); Bifidobacterium lactis BB-12 (109 UFC) } \\
\text { Apresentação: sachê de } 1 \mathrm{~g}\end{array}$ \\
\hline \multirow[t]{7}{*}{ Simbiótico } & $\begin{array}{l}\text { Probiatop (FQM } \\
\text { Farmanutrição) }\end{array}$ & Equilíbrio intestinal & $\begin{array}{l}\text { Lactobacillus paracasei }\left(10^{9} \text { UFC }\right) ; \text { Lactobacillus rhamnosus }\left(10^{9} \text { UFC }\right) ; \text { Lactobacillus } \\
\text { acidophilus }\left(10^{9} \text { UFC }\right) ; \text { Bifidobacterium lactis }\left(10^{9} \text { UFC }\right) \\
\text { Apresentação: sachê de } 1 \mathrm{~g}\end{array}$ \\
\hline & Atillus Flora & Contribui para o equilíbrio da flora intestinal & $\begin{array}{l}\text { Lactobacillus Acidophilus SD } 5221-10^{9} \text { UFC; Lactobacillus rhamnosus SD } 5217 \text { - } 10^{9} \text { UFC } \\
\text {;Bifidobacterium Bifidum SD } 6576-10^{9} \text { UFC ; Frutooligossacarídeo - 5,5 g. } \\
\text { Apresentação: Sachê de } 6 \mathrm{~g}\end{array}$ \\
\hline & $\begin{array}{l}\text { Simbioflora } \\
\text { Farmanutrição) }\end{array}$ & Contribui para o equilíbrio da flora intestinal. & $\begin{array}{l}\text { Lactobacillus acidophilus NCFM; Lactobacillus rhamnosus HN001; Lactobacillus } \quad \text { para } \\
\text { casei LPC-37; Bifidobacterium lactis HN019; Fruto oligossacarideo }(6 \mathrm{~g})\end{array}$ \\
\hline & $\begin{array}{l}\text { Fiber mais flora } \\
\text { (Nestlé) }\end{array}$ & $\begin{array}{l}\text { Pacientes que necessitam de equilíbrio da flora } \\
\text { intestinal }\end{array}$ & $\begin{array}{l}\text { Fibras solúveis e prebióticas }(60 \% \text { Goma Parcialmente Hidrolisada e } 40 \% \text { Inulina })+ \\
\text { Lactobacillus reuteri. Alimento com propriedade funcional. } \\
\text { Apresentação: sachê de } 4,3 \mathrm{~g} \text { de fibra alimentar }\end{array}$ \\
\hline & Lactivos (Moryba) & $\begin{array}{l}\text { Indicado no tratamento e prevenção de desordens } \\
\text { intestinais }\end{array}$ & $\begin{array}{l}\text { Lactobacillus acidophilus La } 14 \text { (probiótico) e goma acácia (prebiótico), é enriquecido com } \\
\text { vitaminas B1, B2, B6 e C e minerais (cálcio e magnésio). } \\
\text { Apresentação: sachê de } 7 \mathrm{~g}\end{array}$ \\
\hline & Sunbiotics & $\begin{array}{l}\text { Restaurar um equilíbrio adequado da flora no } \\
\text { sistema digestivo esgotado pelo estresse, } \\
\text { antibióticos e distúrbios gastrointestinais. }\end{array}$ & $\begin{array}{l}\text { Mistura de probióticos - } 20 \text { bilhões de UFC (Lactobacillus Acidophilus; Bifidobacterium Bifidum; } \\
\text { Bifidobacterium Lactis; Bifidobacterium Longum; FOS (1200 mg). } \\
\text { Apresentação: Lata de } 57 \mathrm{~g}\end{array}$ \\
\hline & LactoFos (FMQ) & $\begin{array}{l}\text { Efeito benéfico no organismo, reconstituindo e } \\
\text { reequilibrando a flora intestinal de forma natural. }\end{array}$ & $\begin{array}{l}\text { Frutooligossacarídeos - 6g; Lactobacillus casei }-10^{9} \text { a } 10^{6} \text { UFC; Lactobacillus rhamnosus }-10^{9} \text { a } \\
10^{6} \text { UFC; Lactobacillus acidophilus - } 10^{9} \text { a } 10^{6} \text { UFC; Lactobacillus bifidum }-10^{9} \text { a } 10^{6} \text { UFC. } \\
\text { Apresentação: sachê } 6 \mathrm{~g}\end{array}$ \\
\hline
\end{tabular}

Fonte: Sites e aplicativos de empresas especializadas em produtos para nutrição enteral. 
A partir dos dados analisados foram identificadas onze fórmulas com indicação para a CI e para os transtornos gastrointestinais, sendo, uma do laboratório Prodiet, duas Nestlé, quatro Fresenius, duas Danone/Nutrimed e duas do laboratório Abbott. Observou-se que, além das fórmulas nutricionais, foram encontradas 23 insumos suplementares, destes, dez são prebióticos, cinco probióticos e oito simbióticos. Pode-se perceber que dentre os prebióticos as fibras de maior predominância foram fibras solúveis, que segundo Bemaud (2013) a principal característica é ser viscosa ou facilmente fermentável no colón, como: a pectina, goma guar e o polissacarídeo da soja, porém são as fibras insolúveis como: o farelo de trigo, lignina, celulose e hemicelulose que tem ação benéfica na constipação intestinal, pois aumentam o volume do bolo fecal,no entanto este tipo de fibra foi encontradas em pequena variedade, predominando apenas a celulose.

Em relação aos probióticos, foi encontrado uma quantidade menor, e com uma variedade monótona de bactérias na composição, as mais comuns foram: Lactobacillusacidophilus, Bifidobacteriumlactis, Bifidobacterium bifidum e lactobacillus casei, deixando clara a necessidade de uma maior variedade de produtos ricos nestes compostos funcionais que são de grande importância no tratamento da constipação intestinal em TNE, e que podem ser acrescidos em preparações alimentícias, medicamentos e suplementos dietéticos (Krumbecket al., 2016).

Além dos probióticos e prebióticos identificou-se também a presença dos simbióticos, que segundo Trindade (2012) tem uma potencialidade e eficácia bem relevante no tratamento da CI, essa combinação de prebiótico e probiótico aumenta a ação de cada um, intensificando os benefícios e efetividade, proporcionando melhores resultados no tratamento. Desse modo, fica clara a importância de alternativas intervenientes nesta situação clínica e, assim, o Guia foi pensado e elaborado com informações nutricionais inerentes a $\mathrm{CI}$ e com sugestões de fórmulas e suplementos disponíveis no mercado compostos por prebióticos, probióticos e simbióticos, possibilitando facilitar e dar suporte ao manejo adequado na prescrição dietética em TNE de adultos e idosos que requeiram cuidados quanto à constipação intestinal.

O guia contempla estratégias e orientações de promoção à saúde, o material dispõe de uma linguagem simples com uso de imagens para uma melhor comunicação com o leitor, a fim de facilitar a compreensão da mensagem e minimizar as barreiras da comunicação. Caracteriza-se como uma ferramenta eficiente e de maior alcance ao público alvo, buscando ser convidativo, de fácil leitura e entendimento. Todavia um material como esse pode ter algumas limitações no que tange as fórmulas apresentadas que podem com o passar do tempo sofrerem modificações ou entrarem em desuso no mercado, o que seria necessárias atualizações no guia.

\subsection{Fechamento e Referências. (Figura 5)}

No tópico conclusão há uma breve explicação sobre o uso do guia, explicitando que o mesmo não substitui os protocolos existentes para o tratamento da TNE. E as referências apresentam os sites onde se podem encontrar os insumos apresentados ao longo do guia. 
Research, Society and Development, v. 11, n. 2, e48011225801, 2022

(CC BY 4.0) | ISSN 2525-3409 | DOI: http://dx.doi.org/10.33448/rsd-v11i2.25801

Figura 5 .

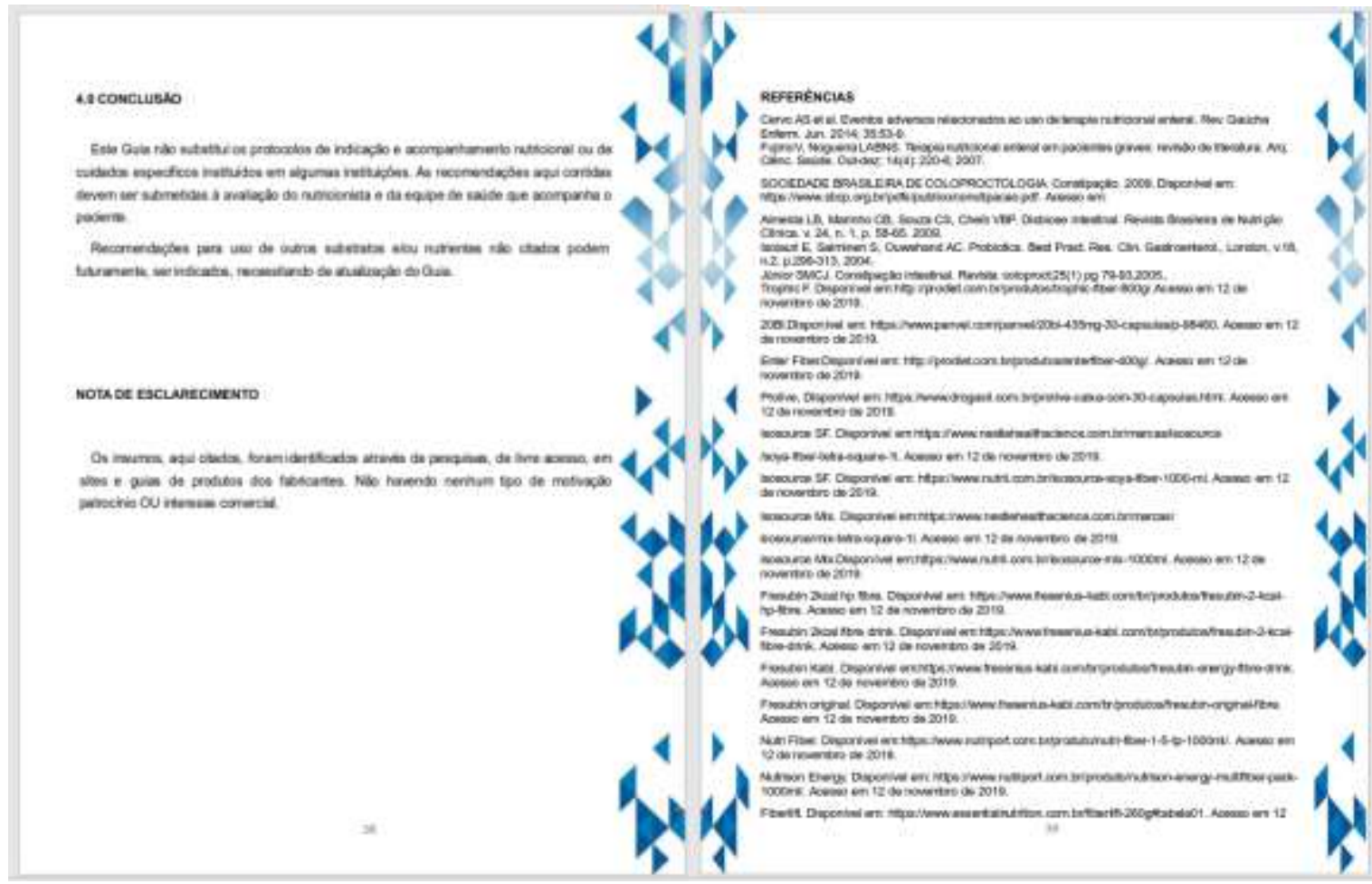

Fonte: Autores.

\section{Conclusão}

O Guia, enquanto tecnologia educacional se configura em uma ferramenta prática e útil que tem o propósito de conduzir os profissionais da área da saúde, sobretudo nutricionistas, na adoção de condutas dietoterápicas adequadas aos pacientes acometidos de CI. Condutas adequadas se fazem necessárias não só pelas variadas etiologias da CI, mas pela escassez de insumos dietéticos disponíveis no mercado. Sugere - se estudos mais amplos que considerem avaliação do estado nutricional dos pacientes e melhora da CI com a prática dos manejos e consumo dos insumos aqui descritos.

\section{Referências}

Almeida, L. B., \& Marinho, C. B., \& Souza, C. S., \& Cheib, V. B. P. (2009). Disbiose intestinal. Belo horizonte: Revista Brasileira de Nutrição Clínica.

Barbosa, R M. S., \& Colares. L. G. T., \& Soares, A. (2008). Desenvolvimento de guias alimentares em diversos países. Rev. Nutr. https://doi.org/10.1590/S1415-52732008000400010

Barros, R. J., \& Soares, M. F., \& Lobo, F. M. I. (2019). Incidência de constipação intestinal em uma unidade de terapia intensive. Sergipe: Rev.Nutr. clín. diet. Hosp, 39(2): 80-83 10.12873/392josiane

Bernaud, R. S. F., \& Rodrigues, C. T. (2013). Fibra alimentar: ingestão adequada e efeitos sobre a saúde do metabolismo. Arq Bras Endocrino Metab.

Cervo, A. S. et al. (2014). Eventos adversos relacionados ao uso de terapia nutricional enteral. Rev. Gaúcha Enferm. 35(2):53-9 DOI: http://dx.doi.org/10.1590/1983- 1447.2014.02.42396

Deheinzelin, D. (2012). Redação e literatura científica: Introdução ou por que os seus dados são importantes.: Rev Assoc Med Bras.

Ferreira, C. L. L. F. (2012). Prebióticos e Probióticos: Atualização e prospecção.: Rubio.

Ferreira, G. S. (2014). Disbiose intestinal: Aplicabilidade do probiótico e dos prebioticos na recuperação e manutenção da microbiota intestinal. Palmas.

Guerra, S. L. T., \& Mendonça, S. S., \& Marshall, G. N. (2013). Incidência de constipação intestinal em uma unidade de terapia intensiva. Brasília: revista Brasileira Terapia intensiva.

Isolauri, E., \& Salminen, S., \& Ouwehand, A. C. (2004). Probiotics. Best Pract. Res. Clin. Gastroenterol. 
Research, Society and Development, v. 11, n. 2, e48011225801, 2022

(CC BY 4.0) | ISSN 2525-3409 | DOI: http://dx.doi.org/10.33448/rsd-v11i2.25801

Júnior, S. M. C. J. (2005). Constipação intestinal. Revista colo proct.

Krumbeck, J. A., \& et al. (2016). Prebiotics and synbiotics: dietary strategies for improving gut health. Lincoln: Faculty Publications in food Science and technology.

Maciel, P. I. F. (2002). As cartilhas e as histórias de alfabetização no Brasil: Alguns Apontamentos, história de educação. Pelotas.

Melo, D. R. T., \& et al. (2017). Adequação do suporte nutricional em pacientes em uso de terapia nutricional enteral. Aracaju: revista Nutrição clínica dietética hospitalaria.

Montejo, O., \& Alba, G. et al (2001). Relación entre la viscosidad de las dietas enterales y las complicaciones mecánicas em su administración según el diâmetro de la sonda nasogástrica. Universidad de Barcelona.

Oliveira, J. \& et al. (2021). Aspectos clínicos de pacientes infanto-juvenis em terapia nutricional enteral domiciliar: uma revisão integrative. Rio de Janeiro: Revista de Saúde Coletiva. http://dx.doi.org/10.1590/S0103-73312021310216

Reis, M. A., \& Frchtenicht, V. A., \& Loss, H. S., \& Moreira, F. L. (2018). Uso de fibras em nutrição enteral de pacientes graves: uma revisão sistemática. Revista Brasileira de terapia intensiva.

Santana, J. S., \& Soares, M. J. G. O., \& Nóbrega, M. M. L. (2012). Instument for Visiting in Nursing to Hypertensive Patients in Family: a Methodological Study. Niteroi: Brazilian Journal of Nursing. 10.5935/1676-4285.20113481

Santos, T. T., \& Varavallo, M. A. (2011). A importância de probióticos para o controle e/ou reestruturação da microbiota intestinal. Revista Científica do ITPAC.

Shils, M. E. (2003). Tratado de Nutrição Moderna na Saúde e na doença. Editora Manole.

Silva, N. M., \& Medanha, F. J. (2014). A importância da ferramenta tecnológica no contexto social e educacional. Revista Científica do ITPAC.

Sociedade Brasileira de Coloproctologia (2009. 04 de janeiro de 2022). Constipação. https://www.sbcp.org.br/pdfs/publico/constipacao.pdf.

Trindade, E. Z., \& Barbosa, X. O. L., \& Cortez, B. D. (2012). Os benefícios dos simbióticos na melhora da obstipação intestinal crônica. Revista terra e cultura 